\title{
Miofibroma mandibular periosteal sin componente intraóseo: presentación de un caso en paciente pediátrico
}

\author{
Periosteal mandibular myofibroma without intraosseous \\ component: presentation of a case in a pediatric patient
}

\author{
Javier González Bello,* María Luisa López Salgado, ${ }^{\ddagger}$ Arturo Gómez Pedroso Balandrano ${ }^{\S}$
}

\section{RESUMEN}

Introducción: El miofibroma es una lesión neoplásica benigna de células miofibroblásticas de rara aparición en la región maxilofacial, se considera una patología homóloga en solitario de la miofibromatosis que ocurre cuando se identifican múltiples lesiones en un mismo paciente. Objetivo: Se presenta una variedad clínica periosteal no descrita previamente en la literatura del miofibroma mandibular en un paciente pediátrico. Presentación del caso: Se reporta un caso de siete años de evolución en un paciente femenino recibido a los tres años con diagnóstico de miofibroma mandibular periosteal tratada con la enucleación del tumor y la resección del borde óseo mandibular afectado, también se describen los aspectos clínicos, radiográficos e histopatológicos relevantes al manejo y tratamiento de esta enfermedad. Conclusiones: Es importante documentar casos clínicos de características particulares, en lesiones controversiales de difícil diagnóstico para evitar procedimientos agresivos innecesarios.

Palabras clave: Miofibroma mandibular, miofibroma periosteal, patología benigna pediátrica.

\section{ABSTRACT}

Introduction: Myofibroma is a benign neoplastic lesion of myofibroblastic cells of rare appearance in the maxillofacial region, it is considered a homologous pathology in solitary of myofibromatosis that occurs when multiple lesions are identified in the same patient. Objective: A clinical variety not previously described in the literature of mandibular myofibroma in a pediatric patient is presented. Case presentation: A seven-year history of a female patient received at three years of age with a diagnosis of periosteal mandibular myofibroma treated with enucleation of the tumor and the resection of the affected mandibular osseous border is reported, as well as the clinical, radiographic and histopathological aspects relevant to the management and treatment of this disease. Conclusions: It is important to document clinical cases of particular characteristics, even more so in controversial lesions of difficult diagnosis to avoid unnecessary aggressive procedures.

Keywords: Mandibular myofibroma, periosteal myofibroma, benign pediatric pathology.

* Cirujano Maxilofacial egresado del Hospital Regional «Lic. Adolfo López Mateos», ISSSTE, CDMX.

₹ Jefe de Servicio de Cirugía Maxilofacial Pediátrica del Hospital para el Niño y la Niña. Toluca, Estado de México.

$\S$ Jefe de Servicio y titular del Curso del Servicio de Cirugía Maxilofacial del Hospital Regional «Lic. Adolfo López Mateos», ISSSTE, CDMX.

\author{
Correspondencia: \\ Dr. Javier González Bello \\ E-mail: oralsuite@gmail.com
}

Citar como: González BJ, López SML, Gómez PBA. Miofibroma mandibular periosteal sin componente intraóseo: presentación de un caso en paciente pediátrico. Rev Mex Cir Bucal Maxilofac. 2020; 16 (2 y 3): 118-126. https://dx.doi.org/10.35366/97710 


\section{INTRODUCCIÓN}

El miofibroma es una lesión neoplásica benigna de células miofibroblásticas de rara aparición en la región maxilofacial, ${ }^{1}$ se considera una patología homóloga en solitario de la miofibromatosis que ocurre cuando se identifican múltiples lesiones en un mismo paciente (multicéntrica), ${ }^{2}$ aunque esa variante tiene una predilección marcada a vísceras internas como pulmones, páncreas, riñones y el tracto gastrointestinal.

De acuerdo a la literatura, en la región craneofacial el miofibroma se puede encontrar asociado a tejidos blandos de la región oral, cigomática, orbitaria y nasal entre otros, también se ha descrito de forma intraósea en diversos huesos faciales como el maxilar, la mandíbula, el malar y el frontal; ${ }^{3}$ sin embargo, encontrar una reacción periosteal es poco frecuente. ${ }^{4}$ Para su estudio, se pueden dividir en tres tipos: solitario, multicéntrico ${ }^{5}$ y multicéntrico con compromiso visceral, ${ }^{6}$ todos éstos siendo histopatológicamente indistinguibles. ${ }^{7,8}$

Un artículo de revisión de la literatura de 245 pacientes que examinó todos los casos reportados de miofibromas en la región oral mostró una prevalencia de 1.2:1 en relación hombres-mujeres, con una predilección en las primeras décadas de la vida, y cuando éstas tienen un componente óseo se presentan hasta el $85 \%$ en pacientes pediátricos, siento esta patología la más frecuente de los tumores fibrosos de la infancia, ${ }^{9}$ y aunque los casos reportados en pacientes adultos son escasos, ${ }^{10-12}$ su presentación tanto clínica como radiográfica e histopatológica no difiere mucho al de los pacientes pediátricos.

Hablando exclusivamente de su prevalencia en la cavidad oral la mayor cantidad de lesiones se describen en la mucosa móvil como la lengua, mucosa labial y palatina hasta con un $41.3 \%$ de todos los casos, en la mucosa alveolar se presenta un $18.2 \%$ mientras que de manera intraósea un $22.7 \%$ de todos los casos y un $17.8 \%$ de manera inespecífica reportados en la literatura revisada. ${ }^{13}$

Su etiopatogenia es sumamente controversial, incluso desde los primeros reportes realizados por Williams y Schrum en $1951^{14}$ donde fue denominado como «fibrosarcoma congénito», así como por Sir Arthur Stouth ${ }^{15}$ en 1954 como una «fibrosis congénita generalizada" se reconocía a esta entidad como un fenómeno de comportamiento biológico complejo; en estudios recientes, diversos autores proponen hasta tres posibles teorías para la génesis del miofibroma, una traumática, ${ }^{15}$ una neoplásica ${ }^{16,17}$ y finalmente una en la cual existe asociación a algún folículo dental.3,13 Sin embargo, se reconoce que su incidencia familiar es sumamente baja, $8,13,18,19$ por lo cual son más importantes otros factores en su histopatología que únicamente los genéticos en su etiología. 8,17,18,20

Su comportamiento clínico es variable ${ }^{21}$ desde masas firmes de color rojo a rosado que pueden crecer tanto que interfieren en la oclusión e incluso producen obstrucción de la vía aérea ${ }^{22,23}$ a lesiones totalmente asintomáticas de crecimiento lento y componentes intraóseos clínicamente difíciles de percibir, aunque en estos casos los pacientes pueden desarrollar parestesia, desplazamiento de folículos dentales y/o expansión cortical. ${ }^{5}$ Algunas lesiones son fácilmente resecables, otras se encuentran firmemente adheridas a las estructuras adyacentes. ${ }^{24}$ Por su potencial multicéntrico, se recomienda hacer un rastreo tomográfico completo con foco en los órganos internos, sobre todo en pacientes de temprana edad; ${ }^{3}$ es destacable comentar que no todas las lesiones se podrían presentar en el mismo tiempo clínico, por lo tanto es necesario guardar un seguimiento estricto del paciente a largo plazo..$^{5,19}$

En las radiografías panorámicas pueden presentarse como radiolucidencias moderadamente definidas en la zona anterior o posterior de la mandíbula con un borde esclerótico parcialmente desarrollado ${ }^{2} y$ frecuentemente relacionadas a órganos dentales, las cuales suelen apreciarse como una lesión unilocular bien delimitada que aparenta un tumor de origen odontogénico. ${ }^{25}$ En la resonancia magnética suelen verse fuertemente remarcadas por el contraste de gadolinio. ${ }^{16}$

Su comportamiento clínico en conjunto con estudios de imagen donde haya asociación a órganos dentales puede mimetizar a lesiones como el ameloblastoma, el queratoquiste odontogénico, el quiste dentígero y los tumores de células gigantes, entre otros $^{10}$ y cuando presentan un crecimiento rápido pueden ulcerarse, ocasionando reabsorción dental con perforación de placas corticales aparentando un comportamiento maligno. ${ }^{26}$

Macroscópicamente, estas lesiones se observan como masas grises, blancas o rosas de apariencia gomosa y agrietada, al corte se observan lóbulos fibrosos en su espesor. ${ }^{9}$

Su histología revela un patrón de zonificación nodular bifásico de miofibroblastos compuesto por áreas de tinción variable, donde es frecuente encontrar células espinosas eosinofílicas con un citoplasma claro abundante de núcleos elongados y con cromatina vesicular en la periferia de la 
lesión, en adición se observan pequeñas y densas áreas oscuras de alta celularidad que consisten en miofibroblastos con un citoplasma intensamente eosinofílico de forma redonda a poligonal de núcleos vesiculados. La presencia de estos dos patrones histológicos de cambio abrupto ${ }^{27}$ crea un fenómeno conocido como de «zonificación» distintivo. ${ }^{25,28}$ Con frecuencia es posible observar vasos sanguíneos hemangiopericitomales, estas lesiones suelen tener nula o baja actividad mitótica no relevante en su pronóstico. $^{9}$

Para estas lesiones, el uso de pruebas de inmunohistoquímica es prácticamente obligatorio para corroborar el diagnóstico, así entonces el miofibroma es característicamente negativo a las pruebas de inmunorreactividad CD34, CD99, S-100 y desmina, ${ }^{29}$ mientras que es constantemente positivo a las pruebas de SMA-actina para músculo liso, actina músculo-específica y vimentina las cuales permiten diferenciarla de lesiones como el hemangiopericitoma, mientras que las pruebas para CD34 y S-100 la diferencian de tumores de origen fibroso o neurogénico. ${ }^{12}$

El diagnóstico diferencial de los miofibromas incluye tanto lesiones benignas como sarcomas de bajo grado, radiográficamente, si no hay asociación a órganos dentales cuando las lesiones se localizan de manera intraósea y llegan a exhibir un crecimiento rápido y lítico, pueden incluirse dentro de los diagnósticos diferenciales a lesiones como rabdomiosarcoma, histiocitosis X, neuroblastoma metastásico o fibrosarcoma. ${ }^{19,30}$ Histopatológicamente, los diagnósticos diferenciales pueden incluir leiomiomas intraóseos, fibromatosis y fibrosarcomas, pero difieren por la falta de actividad mitótica, su apariencia mioide y el fenómeno de «Zonificación». ${ }^{16}$

Tanto el diagnóstico presuntivo como el definitivo suelen ser difíciles y controversiales debido a la falta de especificidad de su presentación clínica y radiográfica, asimismo, las similitudes histopatológicas con una gran cantidad de lesiones neoplásicas de células espinosas hacen difícil plantear un tratamiento quirúrgico definitivo, por lo cual aún en la actualidad no existe un «estándar de oro» para el tratamiento de estas lesiones; sin embargo, la mayoría de los autores proponen una escisión quirúrgica conservadora como una biopsia por enucleación y/o el curetaje. ${ }^{8,19}$ Se ha reconocido que el riesgo de recurrencia puede ser de entre el $7^{3}$ al $31 \%^{22}$ de todos los casos, pero esto es cuando se ha eliminado de forma incompleta la lesión, generalmente por inaccesibilidad anatómica ${ }^{31,32}$ y el tratamiento subsecuente suele responder bien al curetaje de la lesión. ${ }^{4}$

Se ha propuesto también el uso de agentes quimioterapéuticos para esta patología ${ }^{23}$ para aquellos casos donde es imposible acceder quirúrgicamente a la lesión o se encuentra multicéntrica, como el caso de la miofibromatosis infantil siguiendo un protocolo trisemanal de vincristina y actinomicina D por seis meses, no obstante, no se han establecido los criterios específicos para esta modalidad de tratamiento y su uso aún es discutible.

Se realizó una extensa revisión de la literatura de los reportes en idioma inglés y español con bases de datos como: PubMed, ScienceDirect y Web of Science usando las palabras clave «miofibroma» «miofibroma intraóseo», «miofibroma mandibular» y «miofibroma facial», cabe destacar que se evitó incluir en el estudio otras nomenclaturas utilizadas para denominar esta patología para evitar errores diagnósticos con otras entidades patológicas.

En general, no existen reportes en la literatura bien descritos del miofibroma presentado en la mandíbula como una agresiva reacción periosteal sin un componente intraóseo franco, por lo cual se realizó la revisión extensa de los casos publicados, así como la presentación de nuestra experiencia con un paciente de siete años de evolución.

\section{PRESENTACIÓN DEL CASO CLÍNICO}

Se trata de un paciente femenino de tres años, quien acude al Servicio de Cirugía Maxilofacial referida del Servicio de Cirugía Pediátrica por presentar un aumento de volumen submandibular izquierdo de dos meses de evolución de acuerdo con los padres, quienes negaron algún antecedente de trauma, fiebre, pérdida de peso u otros síntomas constitucionales (Figuras $1 \mathrm{~A}-\mathrm{C}$ ).

La paciente nació a término mediante parto normal eutócico, sin alguna patología asociada sin reportes de alergias a medicamentos, asimismo, sin historia familiar de miofibromas, miofibromatosis ni malignidades.

Nunca se reportó dificultad para la masticación, siendo de crecimiento lento pero constante, asintomático al momento de la consulta, sin zonas de paresia ni parestesia, con antecedentes de una biopsia submandibular incisional previa realizada por el Servicio de Cirugía Pediátrica que al estudio histopatológico se reporta como tejido inflamatorio inespecífico. 

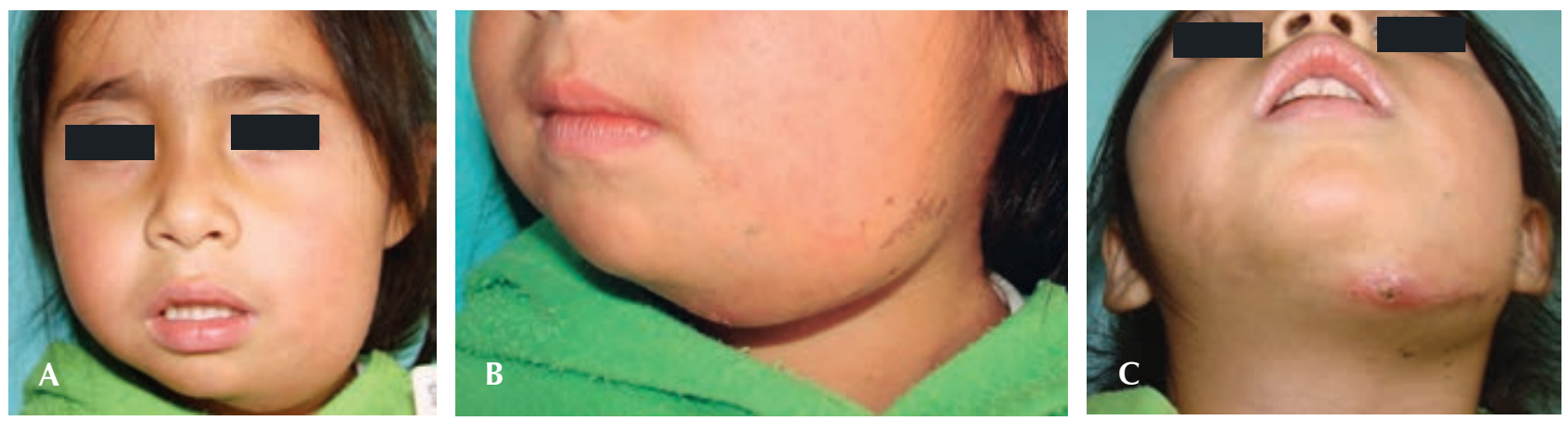

Figura 1: Fotografías iniciales preoperatorias. A) Fotografía de frente donde se aprecia marcada asimetría facial por tumoración en tercio inferior. B) Fotografía de 3/4 donde se observa aumento de volumen submandibular izquierdo. C) Lesión submandibular posterior a la biopsia incisional inicial realizada por Servicio de Cirugía Pediátrica.
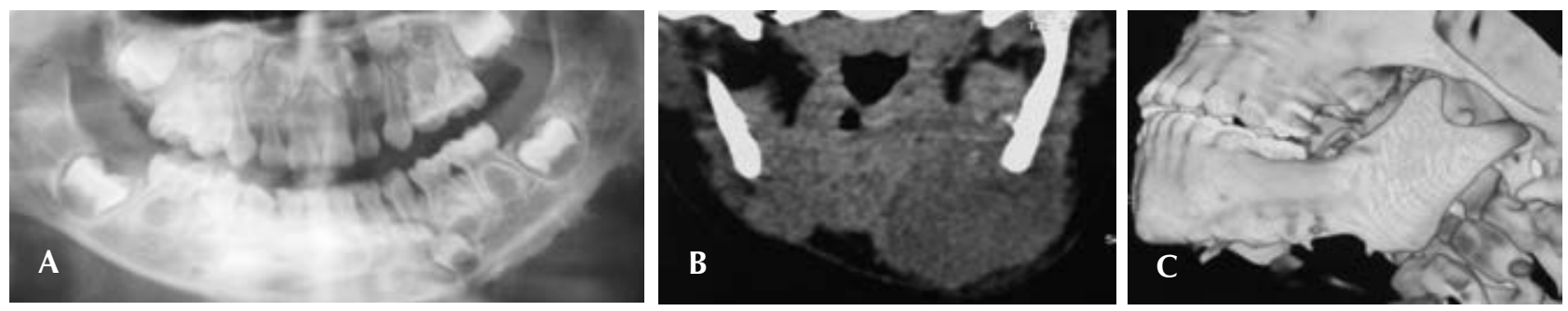

Figura 2: Estudios de imagen preoperatorios. A) Radiografía panorámica donde se observa erosión y asimetría del borde inferior mandibular izquierdo. B) Se observa corte coronal de tomografía computarizada a nivel de cóndilo mandibular donde se aprecia aumento de volumen en borde mandibular inferior sin aparente destrucción ósea. C) Reconstrucción tridimensional donde se aprecian prolongaciones óseas a nivel mandibular inferior izquierdo.
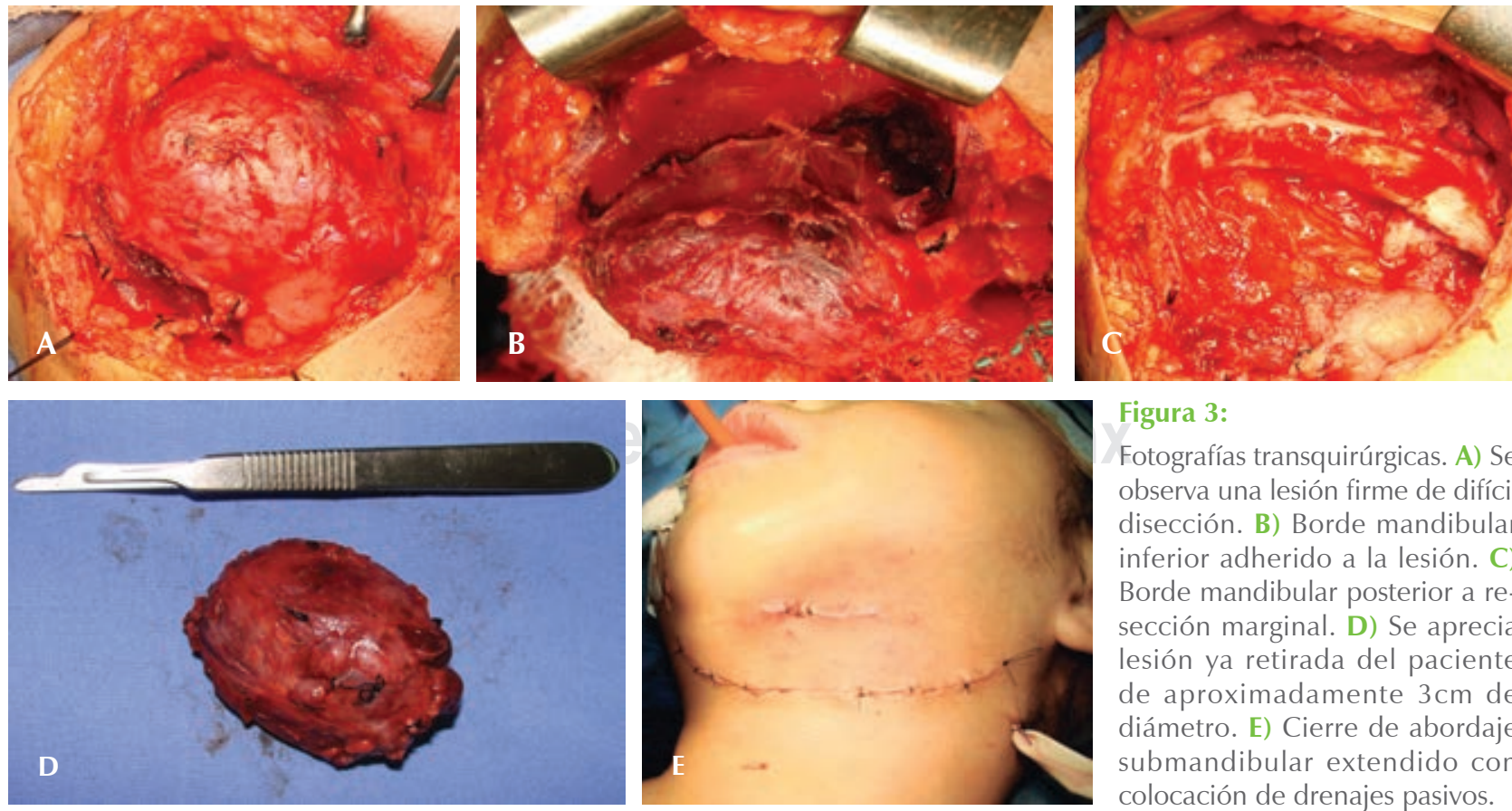

Figura 3:

Fotografías transquirúrgicas. A) Se observa una lesión firme de difícil disección. B) Borde mandibular inferior adherido a la lesión. C) Borde mandibular posterior a resección marginal. D) Se aprecia lesión ya retirada del paciente de aproximadamente $3 \mathrm{~cm}$ de diámetro. E) Cierre de abordaje submandibular extendido con colocación de drenajes pasivos. 
Rev Mex Cir Bucal Maxilofac 2020;16 (2 y 3): 118-126

A la clínica se encuentra un aumento de volumen submandibular izquierdo de aproximadamente $5 \mathrm{~cm}$ de diámetro, con una cicatriz lineal en la zona de aproximadamente $3 \mathrm{~cm}$ correspondiente al sitio de abordaje para la biopsia ya comentada, a la palpación de consistencia firme, fijo a planos profundos y no desplazable, sin evidencia de signos francos de inflamación. A la examinación sistémica no se identificaron otras masas ni linfadenopatías.

La radiografía panorámica (Figura 2A) evidenció una asimetría a expensas del borde mandibular inferior izquierdo, donde se identificó pérdida de la uniformidad en el contorno óseo cortical, con algunas prolongaciones radiopacas más allá del borde mandibular, sin desplazamiento o afección a los órganos dentales ni al trabeculado del hueso.

Se realiza subsecuentemente una tomografía computarizada simple y contrastada en las cuales se aprecia una lesión unilocular asociada al borde inferior izquierdo de la mandíbula, correspondiente a una masa de tejido blando de $3.15 \times 3.27 \mathrm{~cm}$ de diámetro aproximado, desplazando el contorno óseo sin llegar a presentar una invasión medular franca e íntimamente relacionado al periostio, asimismo, se vuelven a observar las prolongaciones óseas provenientes de la cortical mandibular hacia la lesión (Figuras 2 A y B).

Se decide nuevamente una biopsia incisional bajo anestesia general en la cual se emite el diagnóstico de miofibroma, por lo cual la paciente entra en protocolo quirúrgico para biopsia excisional mediante un abordaje submandibular extendido con saucerización del borde submandibular derecho involucrado en la lesión, se realiza la disección de los planos suprayacentes al aumento de volumen sin mayor eventualidad disecando fascia y músculo, pero cabe destacar que no fue posible resecar la lesión del borde óseo submandibular y se encontró franca continuidad al periostio, por lo que se decide realizar una osteotomía limitada a la cortical adyacente (Figuras 3 A-E).

Se obtiene un espécimen de $32 \times 24 \times 15 \mathrm{~mm}$ de superficie lisa a lobulada, color pardo a amarillo aparentemente en su totalidad, el cual al corte se aprecia de un espesor solido amarillo con zonas rosas estriadas, donde en su porción superior se encuentra el fragmento óseo osteotomizado de 18 $\times 4 \times 6 \mathrm{~mm}$ descrito macroscópicamente como de color pardo e irregular (Figuras 4 A-C).

En la sección estudiada, se identificó una lesión de estirpe mesenquimatosa, formada por proliferación multinodular no encapsulada de células fusiformes arregladas en fascículos entrelazados en patrón vertical, las células presentaban amplio citoplasma levemente eosinofílico y anguladas así como multinucleadas, en algunas zonas los fascículos con tendencia a agruparse alrededor de espacios vasculares de pared delgada en la periferia, encontrando tejido fibroso maduro con ocasionales células inflamatorias, así como haces de músculo estriado infiltrado en la lesión hasta el borde de sección en algunas áreas (Figuras $5 \mathrm{~A}$ y D). Se realizó inmunohistoquímica de la lesión para corroborar el diagnóstico siendo positivo para vimentina y antiactina (Figuras 5 B-C).

A la primera semana postoperatoria se presenta la paciente con buena evolución para retiro de drenajes, sin el aumento de volumen, pero con ligera paresia de rama mandibular de nervio facial izquierdo, por lo cual se inicia fisioterapia (Figuras 6 A y B). Se solicita radiografía panorámica de control donde se aprecia borde mandibular inferior con zona de osteotomía sin lesión a gérmenes dentales (Figura 7A).

Se muestra el control de la paciente a siete años de evolución sin evidencia de recidiva clínica ni imagenológica (Figura 7B) conservando adecuada función y simetría facial sin restricción en el crecimiento ni en la longitud mandibular, exhibiendo únicamente un defecto en la zona del borde óseo inferior, el cual será reconstruido una vez haya terminado el crecimiento, con remisión total de la paresia de rama mandibular inferior (Figuras $8 \mathrm{~A}-\mathrm{C}$ ).

\section{DISCUSIÓN}

Los estudios de inmunohistoquímicos para estas lesiones no han sido totalmente elucidados y aún no existe un "estándar de oro» en cuanto a los agentes reactivos específicos para esta patología, aunque en un estudio del 2004 por Parish P. Sedghizadeh y colaboradores se propone a la Calponina como un marcador específico para músculo liso, cuya positividad resulta convincente, ${ }^{2}$ siendo este un marcador específico de adenosín trifosfato de miosina para músculo liso utilizado para identificar expresión de miofibroblastos en patología mamaria.

En la literatura revisada, cuando estas lesiones se presentan de manera solitaria e intraósea lo hacen en la región de la cabeza y cuello principalmente, ${ }^{33}$ cuando se aprecian de manera multicéntrica es frecuente encontrarlas asociadas a tejidos blandos en zonas como abdomen y extremidades. Pocos casos similares existen reportados en donde exista una reacción periosteal tan marcada que clínicamente 
se presenten como masas erosivas adyacentes al tejido oseo. ${ }^{6}$

Foss y Ellis, en el año 2000, describen una serie de 79 casos de miofibromas, de los cuales un tercio de los casos afectaron a los maxilares, es importante resaltar que ellos encontraron que 12 fueron centrales o intramedulares (intraóseos) y 15 con compromiso cortical o periosteal. ${ }^{8}$ Esta presentación cortical fue descrita como lesiones exofíticas de tejido blando que a la radiografía mostraron una cubierta delgada y radiopaca, cubriendo un defecto lítico no corticado de la superficie ósea.
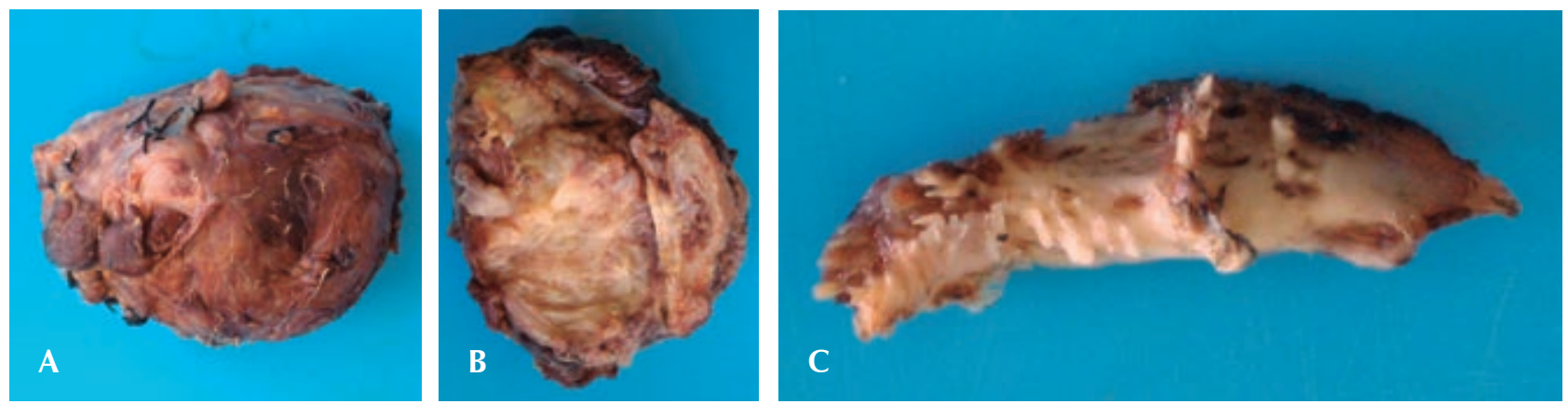

Figura 4: Evaluación macroscópica de la pieza quirúrgica. A) Lesión de $3 \times 3 \mathrm{~cm}$ de superficie lisa de color pardo a amarillo. B) Al corte se aprecia un espesor sólido amarillo con zonas rosas estriadas. C) Se aprecia segmento óseo resecado con múltiples prolongaciones óseas concordantes con lo visto en tomografía.
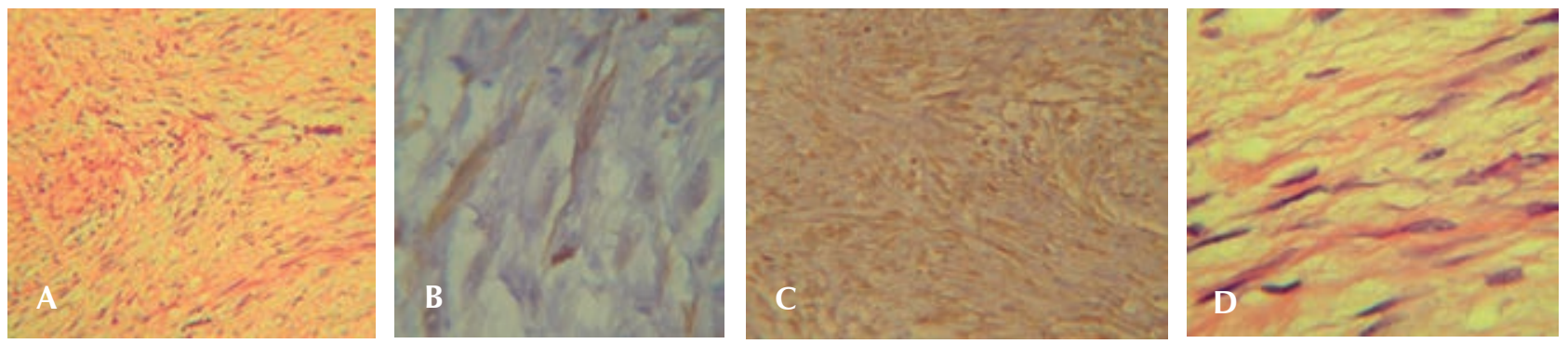

Figura 5: Estudio histopatológico de la lesión. A) Tinción hematoxilina y eosina X40. B) Inmunohistoquímica positiva a antiactina. C) Inmunohistoquímica positiva a Vimentina. D) Tinción hematoxilina y eosina X100.

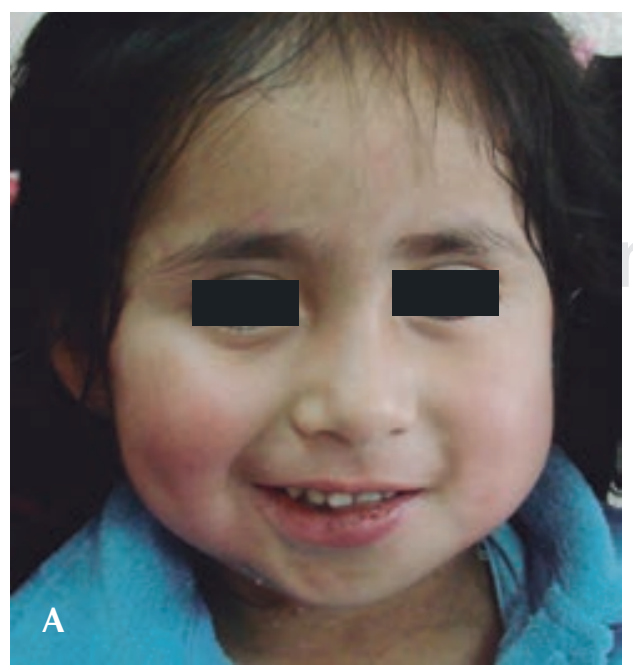

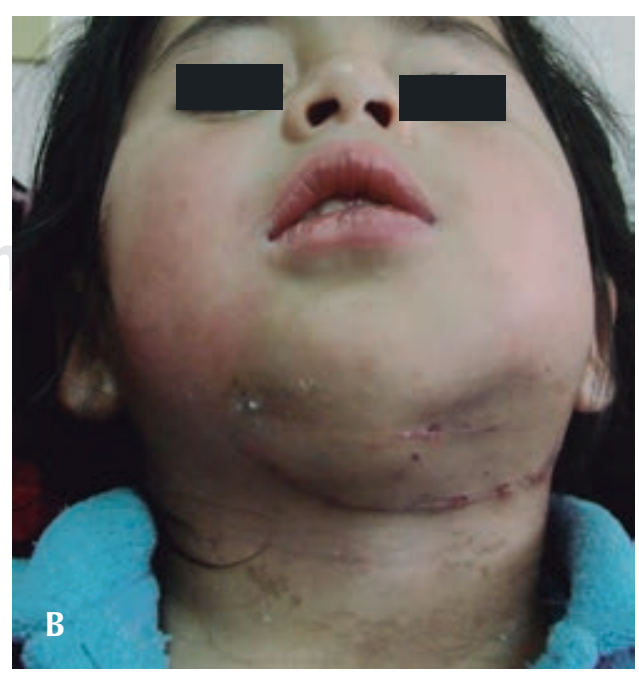

Figura 6:

Postoperatorio mediato a la semana de cirugía. A) Nótese ligera paresia del nervio facial. B) Se observa disminución considerable del aumento de volumen con buena cicatrización. 

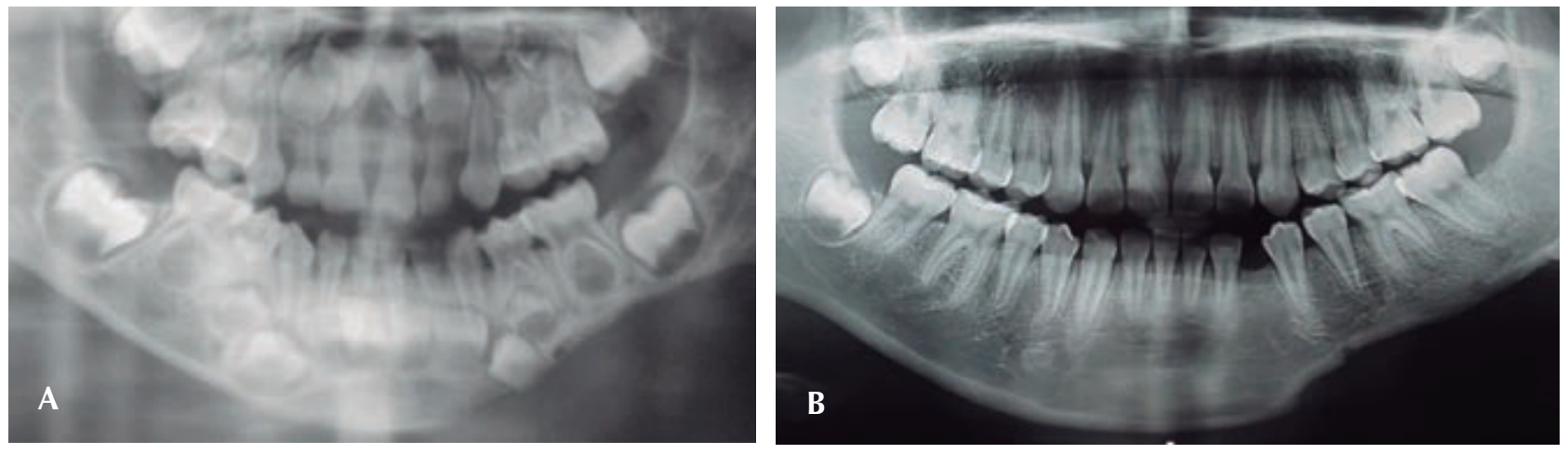

Figura 7: Radiografías postoperatorias panorámicas del paciente. A) Control radiográfico a un mes. B) Control radiográfico a 10 años de evolución.
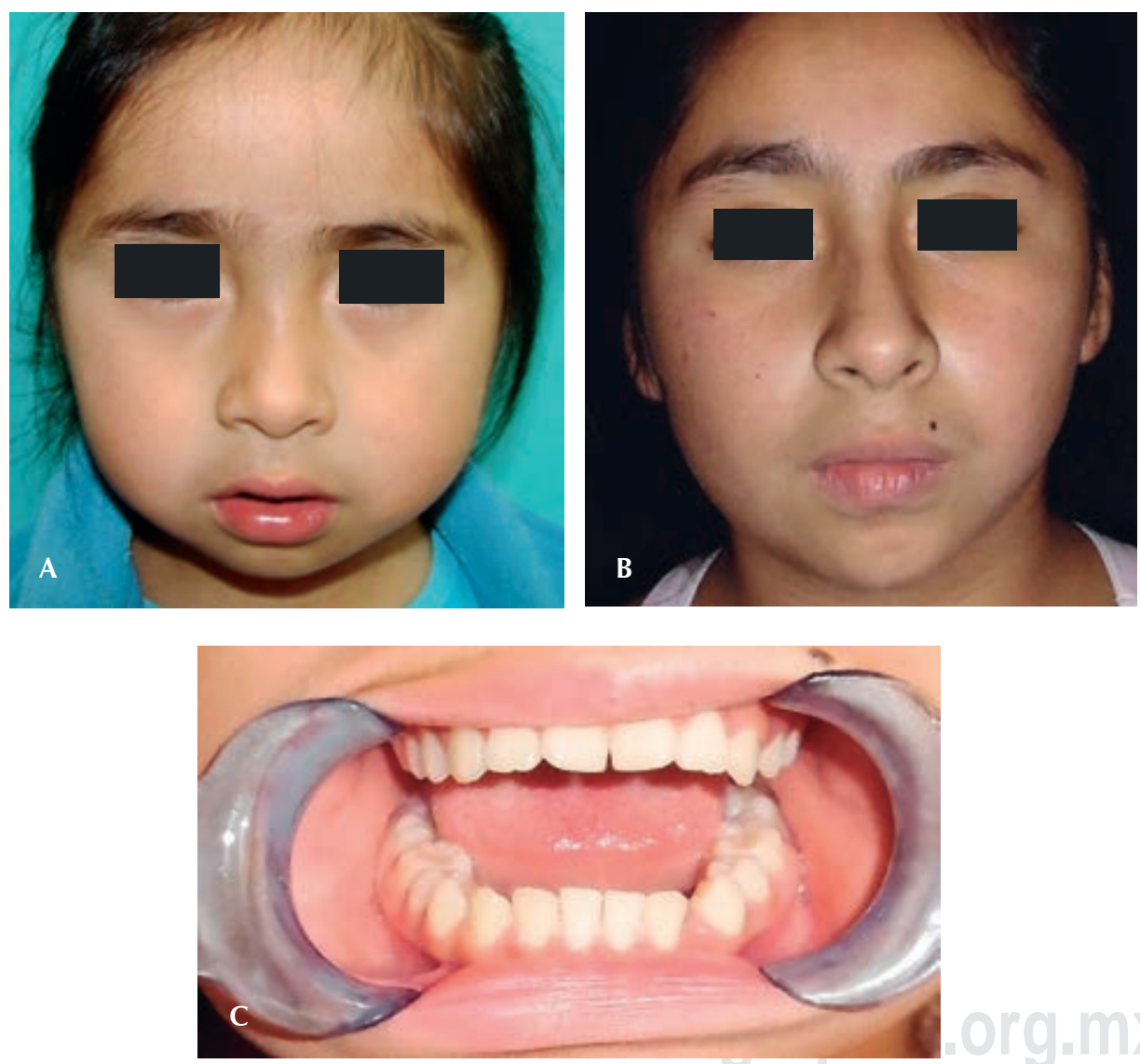

Figura 8:

Fotografías postoperatorias de control. A) Control a un mes de evolución. B) Control a 10 años de evolución sin evidencia de recidiva. C) Control a 10 años con buena apertura oral, sin desviación ni asimetría con alteraciones en el desarrollo óseo.

En el año 2005, un caso descrito como miofibromatosis infantil fue descrito con una lesión solitaria erosionando la eminencia cigomática recubierta por una cápsula aparentemente derivada del periostio; sin embargo, se reporta que esta lesión se disecó fácilmente del hueso adyacente, radiográficamente se muestra de características similares al caso presentado en nuestro trabajo, una masa de tejido blando homogéneo con erosión ósea de la pared anterior del seno maxilar. ${ }^{34}$

Algunos autores creen que en edades tempranas puede existir regresión de esta lesión $n^{5,23}$ mientras que otros refutan esta teoría, ${ }^{22}$ también el miofibroma puede desarrollarse durante la vía intrauterina y 
exhibirse como una lesión relativamente grande al momento del nacimiento ${ }^{5}$ o crecer de manera dramática durante las primeras semanas de vida ${ }^{35}$ o incluso manifestarse años más tarde, como en el caso aquí presentado.

Aunque se han propuesto diversas causas para el miofibroma, la realidad es que su causa exacta aún es desconocida, algunos estudios proponen un origen a partir de restos de miopericitos y células mesenquimales perivasculares, pero no sustentan la reactividad de las mismas en el rol de estas neoplasias. ${ }^{4}$

En realidad, existen pocos casos reportados de miofibroma mandibular bien descritos en la literatura, únicamente 39 hasta el momento, en los cuales es frecuente encontrar asociación a algún folículo u órgano dental, ${ }^{1}$ lo cual refuerza la teoría de un posible origen odontogénico cuando se manifiesta en la región mandibular. ${ }^{25}$ Una constante en la literatura reportada tanto para los tumores intraóseos como en los de tejidos blandos es su fácil disección quirúrgica; sin embargo, en el caso aquí presentado la variante periosteal ameritó la resección del borde óseo al cual se presentaba firmemente adherido en la lesión.

\section{CONCLUSIÓN}

El miofibroma es una neoplasia benigna rara de células espinosas que representa una proliferación de «miofibroblastos», estas células, de acuerdo a su inmunohistoquímica, se describen como fibroblastos y células de músculo liso (miopericítico), esto ha orillado a los investigadores a argumentar que teóricamente no existen células verdaderamente miofibroblásticas, ${ }^{2}$ no obstante, en la práctica resulta innegable su presencia en los cortes histológicos, donde los miofibromas muestran diferenciación miofibroblástica de células espinosas a ovaladas con citoplasma escaso y una organización en fascículos de células, con una morfología más pequeña pero un citoplasma eosinofílico intenso en alternancia formando el característico patrón micronodular conocido como el «fenómeno de zonificación».

Por lo tanto, es importante documentar casos clínicos de características particulares, más aún en lesiones controversiales de difícil diagnóstico, para evitar procedimientos agresivos innecesarios, donde aún se siguen indagando aspectos como su etiopatología o su clasificación e inclusión dentro de otro tipo de neoplasias, tomando en cuenta el seguimiento de recurrencias a largo plazo y la vigilancia del paciente durante los picos de desarrollo.

\section{AGRADECIMIENTOS}

A los cirujanos maxilofaciales, a la Doctora María Luisa López Salgado por permitirnos participar en la elaboración del presente trabajo de investigación y al Doctor Arturo Gómez Pedroso Balandrano por su dedicación, paciencia y profesionalismo en las asesorías recibidas durante la residencia y el presente proyecto.

\section{BIBLIOGRAFÍA}

1. Obine $\mathrm{CH}$, Egg GH, Rossi LT, Batistade FJ, De Andrade MH, Marques $\mathrm{CH}$. Myofibroma of the mandible. Case report. Ped Dent J. 2016; 26 (1): 38-41.

2. Sedghizadeh PP, Allen CM, Kalmar JR, Miloro M, Suster S. Solitary central myofibroma presenting in the gnathic region. Ann Diagn Pathol. 2004; 8 (5): 284-289.

3. Thennavan A, Narayanaswamy V, Niazi TM, Rao L, Radhakrishnan R. Infantile myofibroma eroding into the frontal bone: a case report and review of its histopathologic differential diagnosis. Case Rep Pediatr. 2012; 2012: 630804. doi: 10.1155/2012/630804.

4. Rai B, Ludusan E, McGovern B, Sharif F. Mandibular swelling in a 5-year-old child--mandibular myofibroma. BMJ Case Rep. 2014; 2014. doi: 10.1136/bcr-2014-203977.

5. MacIntosh PW, Grob SR, Stagner AM, Kazlas MA, Jakobiec FA, Yoon MK, Lee NG. Multicentric myofibromatosis presenting as a large congenital eyelid myofibroma. J AAPOS. 2016; 20 (1): 70-73.

6. Eshraghi B, Dehghani S, Saeedi-Anari G. A rare erosive orbital mass in a child: Case report of myofibroma. J Curr Ophthalmol. 2017; 29 (3): 224-227.

7. Brierley DJ, Khurram SA, Speight PM. Solitary myofibroma of the adult mandible: a case report. Oral Surg Oral Med Oral Pathol Oral Radiol. 2013; 115 (3): e40-43.

8. Foss RD, Ellis GL. Myofibromas and myofibromatosis of the oral region: A clinicopathologic analysis of 79 cases. Oral Surg Oral Med Oral Pathol Oral Radiol Endod. 2000; 89 (1): 57-65.

9. LeBlanc RE, Taube J. Myofibroma, myopericytoma, myoepithelioma, and myofibroblastoma of skin and soft tissue. Surg Pathol Clin. 2011; 4 (3): 745-759.

10. Oliver RJ, Coulthard P, Carre C, Sloan P. Solitary adult myofibroma of the mandible simulating an odontogenic cyst. Oral Oncol. 2003; 39 (6): 626-629.

11. Al-Qattan MM, Arafah MM. Six cases of myofibroma--the adult counterpart ofinfantile myofibromatosis: case report. J Hand Surg Am. 2016; 41 (2): 251-256.

12. Saleh RR, Rodic S, Musharrafieh U, Jabbour MN, Sabri A. Myofibroma of the tongue: a case report of a rapidly growing tumor and review of characteristics. Journal of Oral and Maxillofacial Surgery, Medicine, and Pathology. 2015; 27 (3): 422-426.

13. Smith MH, Reith JD, Cohen DM, Islam NM, Sibille KT, Bhattacharyya I. An updateon myofibromas and myofibromatosis affecting the oral regions with report of 24new cases. Oral Surg Oral Med Oral Pathol Oral Radiol. 2017; 124 (1): 62-75.

14. Williams JO, Schrum D. Congenital fibrosarcoma; report of a case in a newborn infant. AMA Arch Pathol. 1951; 51 (5): 548-552.

15. Stout AP. Juvenile fibromatoses. Cancer. 1954; 7 (5): 953-978.

16. Montgomery E, Speight PM, Fischer C. Myofibromas presenting in the oral cavity: a series of 9 cases. Oral Surg Oral Med Oral Pathol Oral Radiol Endod. 2000; 89: 343-348.

17. Jennings TA, Duray PH, Collins FS, Sabetta J, Enzinger FM. Infantile myofibromatosis. Evidence for an autosomal-dominant disorder. Am J Surg Pathol. 1984; 8: 529-538. 
18. Bracko M, Cindro L, Golouh R. Familial occurrence of infantile myofibromatosis. Cancer. 1992; 69: 1294-1299.

19. Scheper MA, Difabio VE, Sauk JJ, Nikitakis NG. Myofibromatosis: a case report with a unique clinical presentation. Oral Surg Oral Med Oral Pathol Oral Radiol Endod. 2005; 99 (3): 325-330.

20. Baird PA, Worth AJ. Congenital generalized fibromatosis: an autosomal recessive condition? Clin Genet. 1976; 9: 488-994.

21. Melikoglu C, Keklik B, Sutcu M, Can N, Aydin A. Infantile myofibroma: A differential diagnosis of hand tumors during the neonatal period. Journal of Pediatric Surgery Case Reports. 2014; 2 (5): 222-224.

22. Garcia-Perla A, Belmonte-Caro R, Infante-Cossio P, MuñozRamos M, Esteban-Ortega F. Upper airway distress due to an oropharyngeal infantile myofibroma. J Craniomaxillofac Surg. 2012; 40 (4): e112-114.

23. Eze N, Pitkin L, Crowley S, Wilson P, Daya H. Solitary infantile myofibroma compromising the airway. Int $\mathrm{J}$ Pediatr Otorhinolaryngol. 2004; 68 (12) 1533-1537.

24. Sugatani T, Inui M, Tagawa T, Seki Y, Mori A, Yoneda J. Myofibroma of the mandible. Clinicopathologic study and review of the literature. Oral Surg Oral Med Oral Pathol Oral Radiol Endod. 1995; 80 (3): 303-309.

25. Sundaravel S, Anuthama K, Prasad H, Sherlin HJ, Ilayaraja V. Intraosseous myofibroma of mandible: a rarity of jaws: With clinical, radiological, histopathological and immunohistochemical features. J Oral Maxillofac Pathol. 2013; 17 (1): 121-125.

26. Allon I, Vered M, Buchner A, Dayan D. Central (intraosseous) myofibroma of the mandible: clinical, radiologic, and histopathologic features of a rare lesion. Oral Surg Oral Med Oral Pathol Oral Radiol Endod. 2007; 103 (4): e45-53.
27. Lingen MW, Mostofi RS, Solt DB. Myofibromas of the oral cavity. Oral Surg Oral Med Oral Pathol Oral Radiol Endodont. 1995; 80 (3): 297-302.

28. Coimbra F, Moreira P, Lopes O. Solitary myofibroma in the oral vestibule. A case report. Revista Portuguesa de Estomatologia. 2008; 49 (2): 93-95.

29. Mbekeani JN, Kemeny JL, Nezzar H. Infantile orbital myofibroma. Int J Pediatr Adolesc Med. 2015; 2 (1): 44-46, ISSN 2352-6467.

30. Azevedo RS, Pires FR, Della CR, de Almeida OP, Kowalski LP Lopes MA. Oral myofibromas: report of two cases and review of clinical and histopathologic differential diagnosis. Oral Surg Oral Med Oral Pathol Oral Radiol Endod. 2008; 105 (6): e35-e40.

31. Liu CJ, Chang KW. "Infantile" myofibroma of the oral cavity: Report of case. Journal of Oral and Maxillofacial Surgery. 2001; 59 (4): 471-472.

32. Josephson GD, Patel S, Duckworth L, Goldstein J. Infantile myofibroma of the nasal cavity; a case report and review of the literature. Int J Pediatr Otorhinolaryngol. 2010; 74 (12): 1452-1454.

33. Natarajan Nirvikalpa, Vinod Narayanan. Intra-osseous infantile myofibroma of the mandible: a case report. In Journal of Oral and Maxillofacial Surgery, Medicine, and Pathology. 2012; 24 (2): 128-131.

34. Franzese CB, Carron J. Infantile myofibromatosis: unusual diagnosis in an older child. Int J Pediatr Otorhinolaryngol. 2005; 69 (6): 865-868.

35. Haspel AC, Coviello VF, Stevens M, Robinson PG. Myofibroma of the mandible in an infant: case report, review of the literature, and discussion. J Oral Maxillofac Surg. 2012; 70 (7): 1599-1604.

\section{Conflicto de intereses: No hay conflicto de intereses.}

